\title{
Adaptive Target Birth Intensity for ET-PHD Filter
}

\author{
Lu Miao, Xin-xi Feng and Luo-jia Chi \\ School of Information and Navigation, Air Force Engineering University, Xi'an, China
}

\begin{abstract}
An adaptive tracking algorithm based on Extended target Probability Hypothesis Density (ETPHD) filter is proposed for extended target tracking problem with priori unknown target birth intensity.The algorithm is implemented by gaussian mixture, where the target birth intensity is generated by measurement-driven, and the persistent and the newborn targets intensity are respectively predicted and updated. The simulation results show that the proposed algorithm improves the performance of the probability hypothesis density filter in the extended target tracking.
\end{abstract}

\section{INTRODUCTION}

Multiple target tracking technology is widely used in sensor networks, machine recognition and positioning, and other fields. Its aim is to continuously predict and update the state of the dynamic target of the number of time varying according to the sensor observation in the clutter environment. Due to the continuous improvement of sensor resolution, more and more attention and research are paid to the extended target tracking problem, and the extended target occupies multiple sensor resolution units at each observation moment.

Traditional multi-target tracking is based on data Association technology, which exists combinatorial explosion and associated uncertainty problem, and is not suitable for multiple extended target tracking. Mahler first uses the Random finite set theory to solve the multitarget tracking problem and proposes the extended target Probability Hypothesis Density (ET-PHD) filter[1]. The existing PHD implementation methods include Gaussian mixture method[2] and particle method[3], and their improved algorithm[4-7].

For the traditional ET-PHD filter, it is assumed that the target birth intensity is a priori known, but in situations where the targets may appear anywhere in the surveillance volume. For this problem, the literature [8] proposed a measurement-driven PHD filter. However, this algorithm only used the measurement location information and ignored the likelihood information. The literature [9] improves on this, and puts forward an adaptive particle PHD filtering algorithm based on measurement-driven. The algorithm is based on the measurement-driven to obtain the target birth intensity, and simultaneously predicts and updates the persistent and the newborn targets. The filtering performance is improved by making full use of the measurement likelihood information.

In this paper, an adaptive extended target tracking algorithm based on ET-PHD filter is proposed to solve the extended target tracking problem with priori unknown target birth intensity. The target birth intensity was obtained by adaptive updating of the measured information obtained by each scan, so as to solve the dependence on the priori hypothesis. In the process of filtering, the persistent and the newborn targets are predicted and updated simultaneously. The simulation results show that the proposed algorithm improves the stability of the extended target number estimation and has better state estimation accuracy.

\section{ADAPTIVE ET-PHD FILTERING ALGORITHM AND GAUSSIAN MIXTURE IMPLEMENTATION}

\subsection{Adaptive ET-PHD filtering algorithm}

In time $\mathrm{k}$, the extended target is divided into two categories: persistent target and newborn target, and differentiated by label $\beta$.

$$
\beta=\left\{\begin{array}{l}
0, \text { persistent } t \arg e t \\
1, \text { newborn } t \arg e t
\end{array}\right.
$$

Make the following assumptions:

Assumption 1: The extended target measurements obeys Poisson distribution and is independent of the target state, i.e., $\gamma(\cdot)=\gamma$;

Assumption 2: The target survival and detection probability are independent with target state, i.e., $P_{S, k}(\cdot)=P_{S}$ and $P_{D, k}(\cdot)=P_{D}$;

Assumption 3: The clutter intensity obeys the Poisson distribution and is uniformly distributed in the observed region.

In time $\mathrm{k}$, the prediction of ET-PHD filter with unknown new target intensity is as follows:

*Corresponding author: 1766584727@qq.com, fengxinxi2005@aliyun.com,592255820@qq.com 


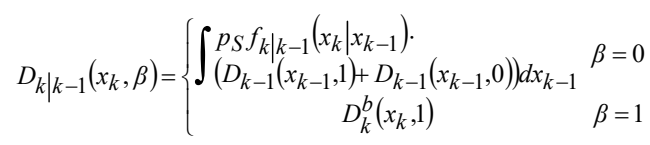

In time $\mathrm{k}$, the posterior PHD is multiplied by predictive PHD and measurement pseudo likelihood function, i.e., $D_{k}\left(x_{k}\right)=L_{Z_{k}}\left(x_{k}\right) D_{k \mid k-1}\left(x_{k}\right)$.

The persistent target is updated to:

$$
\begin{gathered}
D_{k}\left(x_{k}, 0\right)=L_{Z_{k}}\left(x_{k}, 0\right) D_{k \mid k-1}\left(x_{k}, 0\right) \\
L_{z_{k}}\left(x_{k}\right)=1-\left(1-e^{-\gamma\left(x_{k}\right)}\right) P_{D}\left(x_{k}\right)+e^{-\gamma\left(x_{k}\right)} P_{D}(x) . \\
\sum_{p \angle Z_{k}} \omega_{p} \sum_{W \in p} \frac{\gamma\left(x_{k}\right){ }^{W \mid}}{d_{w}} \prod_{z_{k} \in W} \frac{g_{k}\left(z_{k} \mid x_{k}\right)}{\lambda_{k} c_{k}\left(z_{k}\right)}
\end{gathered}
$$

The newborn target is updated to:

$$
D_{k}\left(x_{k}, 1\right)=L_{Z_{k}}\left(x_{k}, 1\right) D_{k \mid k-1}\left(x_{k}, 1\right)
$$

Since the newborn target is obtained from the measurement set, it is always detected, that is, $P_{D}=1$. Its pseudo likelihood function is:

$$
L_{z_{k}}\left(x_{k}, 1\right)=\sum_{p \angle Z_{k}} e^{-\gamma(x)} \cdot \omega_{p} \sum_{W \in p} \frac{\gamma(x)^{|W|}}{d_{w}} \prod_{z_{k} \in W} \frac{g_{k}\left(z_{k} \mid x_{k}\right)}{\lambda_{k} c_{k}\left(z_{k}\right)}
$$
by:

The weight in the formula (4) and (6) are calculated

$$
\begin{aligned}
d_{W}= & \delta|W|, 1 \\
& +D_{k \mid k-1}\left(x_{k}, 0\right)\left[e^{-\gamma} P_{D} \gamma^{|W|} \prod_{\mathrm{z}_{k} \in W} \frac{g_{k}\left(z_{k}^{x} \mid x_{k}\right)}{\lambda_{k} c_{k}\left(\mathrm{z}_{k}\right)}\right] \\
& +D_{k \mid k-1}\left(x_{k}, 1\right)\left[e^{-\gamma \gamma} \gamma^{|W|} \prod_{\mathrm{z}_{k} \in W} \frac{g_{k}\left(z_{k}^{x} \mid x_{k}\right)}{\lambda_{k} c_{k}\left(\mathrm{z}_{k}\right)}\right]
\end{aligned}
$$

In the formula, $\delta_{|W|, 1}$ is the Kronecker function; $p \angle Z_{k}$ represents the partition of the measurement set $Z_{k} ; W$ is non-empty cell in $p ;|W|$ represents the observed number in the dividing unit; $\omega_{p}$ and $d_{w}$ are the weights of $p$ and $W$ respectively; $\lambda_{k}$ is the clutter observation expectation number; and $c_{k}\left(z_{k}\right)$ is the clutter distribution in the monitoring area.

\subsection{Gaussian mixture implementation}

Compared with the particle implementation of the PHD filter algorithm, gaussian mixture is more advantageous in computational efficiency and state extraction because it avoids particle sampling and clustering operations.

Step1. Predict

Suppose the posterior PHD intensity in time k-1 is gaussian mixture, namely:

$$
\begin{aligned}
D_{k-1}\left(x_{k-1}, 0\right) & =\sum_{j=1}^{J_{k-1}^{p}} w_{p, k-1}^{(j)} \mathrm{N}\left(x ; m_{p, k-1}^{(j)}, P_{p, k-1}^{(j)}\right) \\
D_{k-1}\left(x_{k-1}, 1\right) & =\sum_{j=1}^{J_{k-1}^{b}} w_{b, k-1}^{(j)} \mathrm{N}\left(x ; m_{b, k-1}^{(j)}, P_{b, k-1}^{(j)}\right)
\end{aligned}
$$

In the equation, $J_{k-1}$ indicates the number of gaussian component, and $w_{k-1}^{j}, m_{k-1}^{j}$ and $P_{k-1}^{j}$ respectively indicate weight value, mean value and covariance of $\mathrm{jth}$ gaussian component.

The Gauss term used to predict the persistent target in time $\mathrm{k}$ consists of the Gauss term of the persistent target and the newborn target in time k-1, namely:

$$
\begin{gathered}
\left\{w_{k-1}^{(j)}, m_{k-1}^{(j)}, P_{k-1}^{(j)}\right\}_{j=1}^{J_{k-1}}=\left\{w_{p, k-1}^{(j)}, m_{p, k-1}^{(j)}, P_{p, k-1}^{(j)}\right\}_{j=1}^{j_{k-1}^{p}} \\
\cup\left\{w_{b, k-1}(j) m_{b, k-1}^{(j)}, P_{b, k-1}^{(j)}\right\}_{j=1}^{b}
\end{gathered}
$$

In time $\mathrm{k}$, persistent target prediction intensity is expressed as Gaussian mixed form, namely:

$$
\begin{gathered}
D_{k \mid k-1}\left(x_{k}, 0\right)=\sum_{j=1}^{J_{k-1}} w_{p, k \mid k-1}^{(j)} \mathrm{N}\left(x ; m_{p, k \mid k-1}^{(j)}, P_{p, k \mid k-1}^{(j)}\right) \\
w_{p, k \mid k-1}^{(j)}=p_{S} w_{k-1}^{(j)} \\
m_{p, k \mid k-1}^{(j)}=f\left(m_{k-1}^{j}\right) \\
P_{p, k \mid k-1}^{(j)}=Q+F P_{k-1}^{(j)} F^{T}
\end{gathered}
$$

In the formula, $f(\cdot)$ is the state transfer function; $F$ is its Jacobian matrix; $Q$ is the process noise variance.

The target birth intensity is obtained by the measurement drive, which is the inversion of the measurement partition unit. In time $\mathrm{k}$, the predictive intensity of the newborn target is expressed as:

$$
\begin{gathered}
D_{k \mid k-1}\left(x_{k}, 1\right)=\sum_{j=1}^{J_{k \mid k-1}^{b}} w_{b, k \mid k-1}^{(j)} \mathrm{N}\left(x ; m_{b, k \mid k-1}^{(j)}, P_{b, k \mid k-1}^{(j)}\right) \\
J_{k \mid k-1}^{b}=\sum_{p \angle Z_{k}}|p| \\
w_{b, k \mid k-1}^{(j)}=\frac{v_{b, k \mid k-1}}{J_{b, k \mid k-1}^{b}} \\
m_{b, k \mid k-1}=h^{-1}\left(z_{k, j}\right) \\
P_{b, k \mid k-1}=H R H^{T}
\end{gathered}
$$

In the equation, $h(\cdot)$ is the measurement equation; $H$ is its Jacobian matrix; $R$ is the measurement noise variance; $|p|$ represents the number of units $W$ of pth measurement division; and $v_{b, k \mid k-1}$ is the expected number of the newborn target.

Step2. Update

In time $k$, the persistent target intensity update is expressed as:

$$
\begin{gathered}
D_{k \mid k}\left(x_{k}\right)=\sum_{m=1}^{J_{k-1}} w_{p, k}^{(m)} N\left(x ; m_{p, k}^{(m)}, P_{p, k}^{(m)}\right)+ \\
\sum_{p \angle Z_{k}} \sum_{W \in p} \sum_{j=1}^{J_{k-1}} w_{p, k}^{(j)} N\left(x ; m_{p, k}^{(j)}, P_{p, k}^{(j)}\right)
\end{gathered}
$$

The Gauss term of the part not detected in the formula is:

$$
\begin{gathered}
w_{p, k}^{(m)}=\left(1-\left(1-e^{-\gamma}\right) P_{D}\right) w_{p, k \mid k-1}^{(m)} \\
m_{p, k}^{(m)}=m_{k \mid k-1}^{(m)} \\
P_{p, k}^{(m)}=P_{k \mid k-1}^{(m)}
\end{gathered}
$$

The Gauss term of the part detected is: 


$$
\begin{gathered}
w_{k}^{j}=\omega_{p} \frac{e^{-\gamma} p_{D} \gamma^{|W|}}{d_{W}} \times \\
\prod_{\mathrm{Z}_{k} \in W} \frac{N\left(\mathrm{z}_{k}^{W} ; H_{k}^{W} m_{k \mid k-1}^{(j)}, S_{k \mid k-1}^{(j)}\right)}{\lambda_{k} c_{k}\left(\mathrm{z}_{k}\right)} w_{k \mid k-1}^{(j)} \\
m_{k}^{j}=m_{k \mid k-1}^{j}+K_{k}^{j}\left(\mathrm{z}_{k}^{W}-H_{k}^{W} m_{k \mid k-1}^{j}\right) \\
P_{k}^{j}=\left(I-K_{k}^{j} H_{k}^{W}\right) P_{k \mid k-1}^{j} \\
S_{k \mid k-1}^{j}=H_{k}^{W} P_{k \mid k-1}^{j}\left(H_{k}^{W}\right)^{T}+R_{k}^{W} \\
K_{k}^{j}=P_{k \mid k-1}^{j}\left(H_{k}^{W}\right)^{T}\left(S_{k \mid k-1}^{j}\right)^{-1}
\end{gathered}
$$

In time $k$, the newborn target intensity update is expressed as:

$$
D_{k \mid k}\left(x_{k}, 1\right)=\sum_{j=1}^{J_{k}^{b}} w_{b, k \mid k}^{(j)} \mathrm{N}\left(x ; m_{b, k \mid k}^{(j)}, P_{b, k \mid k}^{(j)}\right)
$$

In contrast to the persistent target, the update of the Gauss term of the newborn target intensity function contains only the detection part. The Gauss term formula is:

$$
\begin{gathered}
w_{k}^{j}=\omega_{p} \frac{e^{-\gamma} \gamma^{|W|}}{d_{W}} \times \prod_{\mathrm{Z}_{k} \in W} \frac{w_{b, k \mid k-1}^{(j)}}{\lambda_{k} c_{k}\left(\mathrm{z}_{k}\right)} \\
m_{b, k}^{(j)}=m_{b, k \mid k-1}^{(j)} \\
P_{b, k}^{(j)}=P_{b, k \mid k-1}^{(j)}
\end{gathered}
$$

The weight in the formula (24) and (30) are calculated by:

$$
\begin{gathered}
d_{W}=\delta_{|W|, 1}+\sum_{j=1}^{J_{k-1}} e^{-\gamma} P_{D} \gamma^{|W|} \prod_{\times} \frac{N\left(\mathrm{z}_{k}^{W} ; H_{k}^{W} m_{k \mid k-1}^{(j)}, S_{k \mid k-1}^{(j)}\right)}{\lambda_{k} c_{k}\left(\mathrm{z}_{k}\right)} w_{k \mid k-1}^{(j)} \\
+\sum_{i=1}^{J_{k \mid k-1}^{b}} e^{-\gamma} \gamma^{|W|} \prod_{\times} \frac{w_{b, k \mid k-1}^{(i)}}{\lambda_{k} c_{k}\left(\mathrm{z}_{k}\right)}
\end{gathered}
$$

Step3. Pruning and Merging

The Gauss term of Gaussian mixture algorithm is increasing with time, so it is necessary to prun and merge Gauss terms in order to make calculation feasible. The basic idea is: to keep the Gaussian term with large weight, and to merge the Gaussian term with similar distance.

Step4.Target extraction

Extract the state of the extended target:

$$
\hat{X}_{k}=\left\{m_{k}^{j} \mid w_{k}^{j}>0.5\right\}_{j=1}^{\hat{N}(k)}
$$

\section{Simulation Result and Analysis}

It is assumed that sensor tracks four extended targets in two-dimension plane. The surveillance area is [$1000,1000] \mathrm{m} \times[-1000,1000] \mathrm{m}$ and sampling time $\mathrm{T}=1 \mathrm{~s}$, and observation duration is $100 \mathrm{~s}$. Equations of motion and observation of targets all satisfy linear Gaussian model. The extended target motion and the observational equations are:

$$
\begin{gathered}
X_{k}=\left[\begin{array}{llll}
1 & 0 & T & 0 \\
0 & 1 & 0 & T \\
0 & 0 & 1 & 0 \\
0 & 0 & 0 & 1
\end{array}\right] X_{k-1}+\left[\begin{array}{cc}
T^{2 / 2} & 0 \\
0 & T^{2} / 2 \\
T & 0 \\
0 & T
\end{array}\right]\left[\begin{array}{l}
w_{1, k} \\
w_{2, k}
\end{array}\right] \\
Z_{k}=\left[\begin{array}{llll}
1 & 0 & 0 & 0 \\
0 & 1 & 0 & 0
\end{array}\right] X_{k}+\left[\begin{array}{c}
R_{1, k} \\
R_{2, k}
\end{array}\right]
\end{gathered}
$$

The process noise $\left[w_{1, k}, w_{2, k}\right]^{T}$ and observation noise $\left[R_{1, k}, R_{2, k}\right]^{T}$ are zero-mean Gaussian white noises, in which the standard difference of the process noise component $\sigma_{w_{1}}=\sigma_{w_{2}}=2 \mathrm{~m} / \mathrm{s}^{2}$, and the observed noise component standard difference $\sigma_{R_{1}}=\sigma_{R_{2}}=10 \mathrm{~m}$. The probability of survival is set to $\mathrm{P}_{\mathrm{s}, \mathrm{k}}=0.99$, and the probability of detection is $\mathrm{P}_{\mathrm{D}, \mathrm{k}}=0.99$. The extended target measurements and the clutter all obey the Poisson distribution with the mean value of 10 , the Gaussian term pruning threshold $\mathrm{T}_{\mathrm{p}}=10^{-4}$, the fusion threshold $\mathrm{U}=4$, and the maximum Gaussian term $\mathrm{J}_{\max }=100$.

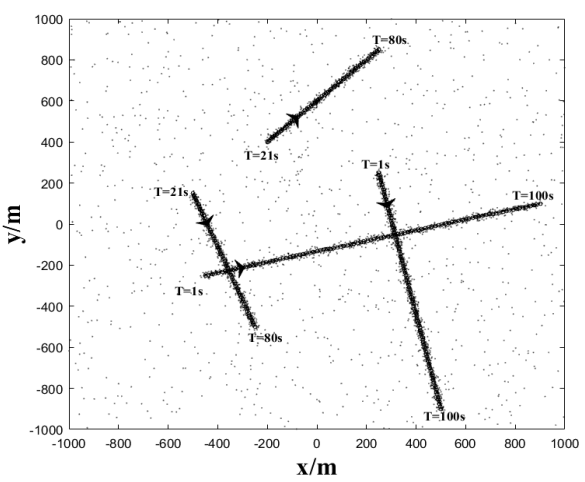

Figure 1: Motion trail of targets in clutter environment.

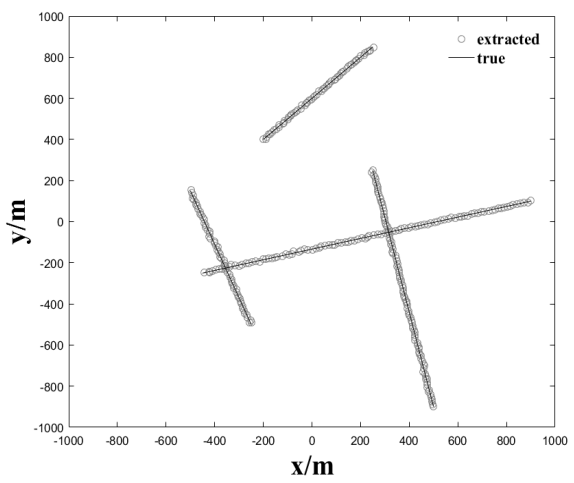

Figure 2: Result of filtering.

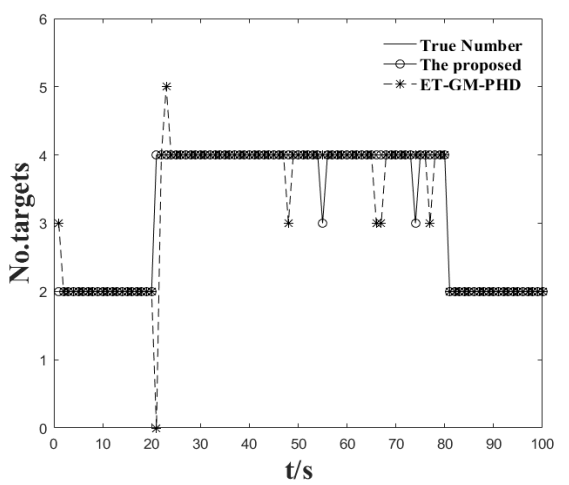

Figure 3: Target quantity estimation. 
Motion trail of extended targets in clutter environment and result of filtering are shown in Fig. 1 and Fig. 2, and target quantity estimation is shown in Fig. 3. It is shown that the proposed algorithm can be used to adaptively design the target birth intensity and realize the effective tracking of the extended targets.

50 times of Monte-Carlo simulation can be conducted to compare the improved algorithm and the original algorithm. We evaluate the performance of the algorithm through the target number estimation, OSPA distance $^{[10]}(\mathrm{p}=2, \mathrm{c}=80)$ and the average computation time. It is shown from table 1 that the improved algorithm can improve the accuracy of target number estimation and has better state estimation result than the original ETGM-PHD filtering algorithm, because the persistent and the newborn targets intensity are respectively predicted and updated, which allows it sufficiently take full advantage of the measurement likelihood information.

Table 1 Filter Performance Comparison

\begin{tabular}{|c|c|c|c|}
\hline $\begin{array}{c}\text { Filtering } \\
\text { algorithm }\end{array}$ & $\begin{array}{c}\text { Target number } \\
\text { estimate } \\
\text { accuracy rate }\end{array}$ & $\begin{array}{c}\text { Average } \\
\text { ospa/m }\end{array}$ & $\begin{array}{c}\text { Average } \\
\text { calculated } \\
\text { time/s }\end{array}$ \\
\hline $\begin{array}{c}\text { Improved } \\
\text { algorithm }\end{array}$ & $97.3 \%$ & 11.3 & 1.10 \\
\hline $\begin{array}{c}\text { Original } \\
\text { algorithm }\end{array}$ & $94.1 \%$ & 15.8 & 1.05 \\
\hline
\end{tabular}

\section{Conclusions}

In this paper, an adaptive extended target PHD filter based on measurement-driven birth intensity is proposed. The algorithm uses the measurements to drive target birth intensity function so as to relax the previously imposed limitation that target birth intensity is a priori known, and the persistent and the newborn targets intensity are respectively predicted and updated to make full use of measurement-likelihood information. The simulation results show that the proposed algorithm improves the estimation accuracy of both the number of targets and target status.

\section{References}

1. R. Mahler. PHD Filters for Nonstandard Targets, I: Extended Targets[C]. In: Proceedings of the 12th International Conference on Information Fusion. USA:IEEE, 2009: 915-921.

2. K. Granstrom, C. Lundquist, U. Orguner. Extended Target Tracking Using a Gaussian-Mixture PHD Filter[J]. IEEE Trans. Aerosp. Electron. Syst., 2012, 48(4): 3268-3286.

3. Y. Li, H. Xiao, Z Song, et al. A new Multiple Extended Target Tracking Algorithm Using PHD Filter[J]. Signal Process, 2013, 93(12): 3578-3588.

4. C. Lundquist, K. Granstrom, U. Orguner. An extended target CPHD Filter and a Gamma Gaussian Inverse Wishart Implementation[J]. Journal of Selected Topics in Signal Processing, 2013, 7(3): 472-483.
5. M. Baum, U. D. Hanebeck. Random Hypersurface Models for Extended Object Tracking[C]. IEEE International Symposium on Signal Processing and Information Technology. IEEE, 2010: 178-183.

6. K. Granstrom, U. Orguner. On spawning and combination of extended/group targets modeled with random matrices[J]. IEEE Transactions on Signal Processing, 2013,61(3): 678-692.

7. N. Wahlström, E. Ozkan. Extended Target Tracking Using Gaussian Processes[J]. IEEE Transactions on Signal Processing, 2015, 63(16): 4165-4178.

8. M. Tobias, A. Lanterman. Techniques for birthparticle placement in the probability hypothesis density particle filter applied to passive radar[J]. IET Radar, Sonar \& Navigation, 2008,2(5): 351-365.

9. B. Ristic, D. Clark, B. N. Vo, et al. Adaptive target birth intensity for PHD and CPHD filters[J]. IEEE Transactions on Aerospace and Electronic Systems. 2012, 48(2): 1656-1668.

10. B. Ristic, B. N. Vo, D. Clark, et al. A Metric for Performance Evaluation of Multi-target Tracking Algorithms[J]. IEEE Trans. Signal Process., 2011, 59(7): 3452-3457. 